\title{
El problema del abandono en estudios a distancia. Respuestas desde el Diálogo Didáctico Mediado
}

\section{(The problem with distance education dropout rate. Answers from the Mediated Educational Dialogue)}

\author{
Lorenzo García Aretio \\ UNED (España)
}

DOI: http://dx.doi.org/10.5944/ried.22.1.22433

\section{Como referenciar este artículo:}

García Aretio, L. (2019). El problema del abandono en estudios a distancia. Respuestas desde el Diálogo Didáctico Mediado. RIED. Revista Iberoamericana de Educación a Distancia, 22(1), pp. 245-270. doi: http://dx.doi.org/10.5944/ ried.22.1.22433

\section{Resumen}

El abandono, el fracaso de los estudiantes universitarios, se muestra como un problema universal que preocupa a docentes, instituciones, gobiernos y organismos internacionales. Esos abandonos, cuando los referimos a los sistemas digitales de enseñanza y aprendizaje (educación a distancia), son aún más elevados que en los formatos presenciales. Los objetivos de este trabajo se centran, por una parte, en seleccionar aquellas causas más destacadas como debilitadoras del diálogo pedagógico (diálogo didáctico mediado) y, en consecuencia, propiciadoras del abandono y, por otra, apuntar acciones orientadoras y de apoyo, que pudieran promover diálogos pedagógicos de calidad que lleven al éxito, a mayores tasas de satisfacción y, en consecuencia, de retención, persistencia y compromiso en los estudios y reducir así el número de fracasos estudiantiles. Procedemos al estudio a través de una revisión de la literatura científica más destacada, para detectar las principales razones que llevan a los estudiantes a distancia al abandono, y una batería de acciones institucionales y docentes que pueden ayudar a reducir esas tasas de fracaso. Y todo ello, centrándolo en la propuesta de Diálogo Didáctico Mediado (García Aretio, 2014). Cuando ese diálogo del estudiante con la institución, con los docentes, los iguales y los recursos, se quiebra, se genera un sentimiento de desánimo, ansiedad y frustración que, si no se remedia, viene a culminar en el abandono de los estudios.

Palabras clave: abandono; deserción; persistencia; retención; éxito; educación a distancia; aprendizaje digital. 


\begin{abstract}
The dropout rate, the failure of university students, appears to be a universal problem that concerns teachers, institutions, governments and international organizations. Dropout rates, when related to teaching and learning through digital systems (distance education), are even higher than in face-to-face formats. The goals of this work focus, firstly, on selecting the most outstanding causes that weaken the pedagogical dialogue (mediated educational dialogue) and, consequently, increase dropout rates, and secondly, on pointing out support and guidance actions that could promote quality pedagogical dialogues leading to success, higher satisfaction rates and, consequently, retention, persistence and academic commitment and, thus, reducing the number of student failures. We proceed to the study through a review of the most outstanding scientific literature to detect the main reasons that motivate distance students to drop out from their studies and a number of institutional and teaching actions that can help reduce those failure rates. The focus here is on the proposal of Mediated Educational Dialogue (García Aretio, 2014). When a student's dialogue with the institution, teachers, peers and resources breaks down, a feeling of discouragement, anxiety and frustration appears which, if not addressed, leads to dropout.
\end{abstract}

Keywords: dropout; abandonment; persistence; retention; success; distance education; elearning.

El diálogo en esta sociedad digital penetra todo tipo de relaciones sociales (Flecha, Gómez y Puigvert 2001) y la educación es ejemplo esencial de relación social. Con esa base, el Diálogo Didáctico Mediado (DDM) es un constructo que, desde la década de los años 90 (García Aretio, 1992, 2001, 2011 y 2014), venimos perfilando para tratar de describir, explicar, comprender y predecir mejor las modalidades educativas a distancia con el fin de regular, con eficacia las intervenciones pedagógicas en ese ámbito. Partimos del supuesto de que en entornos educativos se establecen relaciones sociales que podrían orientarse hacia el diálogo y la participación de los diferentes agentes o, por el contrario, hacia la imposición y/o la inhibición de éstos (Prieto y Duque, 2009). Naturalmente, en este segundo caso, es dudoso que pudiera hablarse de educación.

El diálogo pedagógico, el diálogo didáctico, como conversación, colaboración, cooperación, interacción, intercambio y negociación, se convierte en el protagonista, al relacionar los diferentes componentes y procesos existentes en los sistemas a distancia, hoy, generalmente, sistemas de enseñanza y aprendizaje digitales. En estos entornos en red, de forma más determinante, el aprendizaje, que es un fenómeno social por naturaleza, se construye a través de diferentes diálogos o situaciones conversacionales. La teoría conversacional (Pask, 1975; Scott, 2001), considerada como interacciones múltiples entre las partes involucradas que construyen conocimiento, apoya nuestra perspectiva al afirmar que el aprendizaje se genera a través de múltiples interlocuciones y conexiones humanas y no humanas. De ahí, 
partimos de entender a la educación a distancia, en cualesquiera de sus modalidades y presentaciones como un entramado comunicativo con finalidad pedagógica, donde el estudiante ha de establecer diálogo a través, de los diferentes recursos, con institución y docentes, generalmente ubicados en espacio diferente al de aquél, con el fin de facilitar aprendizajes autónomos y colaborativos (García Aretio, 2014).

Estas relaciones dialógicas comportan diferentes modalidades según los agentes y componentes que interactúan. Así podemos considerar diálogos bidireccionales entre estudiantes-institución, estudiantes-docentes, estudiantes-medios (materiales y vías de comunicación) y estudiantes-estudiantes, y diálogos multidireccionales uno-muchos, muchos-muchos. Además, esos diálogos pueden ser: simulados (por ejemplo, con los materiales de estudio) y reales, síncronos (en directo) y asíncronos (en diferido), unidireccionales y bidireccionales o multidireccionales, verticales y horizontales, estructurados (más rígidos) o flexibles (García Aretio, 2014).

Pues bien, cuando ni siquiera puede iniciarse el diálogo, cuando se produce algún tipo de quiebra en esas relaciones explicitadas, el sistema no fluye adecuadamente o muestra debilidades que podrían llegar a la ruptura final del estudiante con la institución. Es decir, puede llegarse al fracaso del proyecto y, en consecuencia, al drama del abandono o deserción de los estudios y, lamentablemente, esta circunstancia del fracaso de los estudiantes a nivel universitario es más frecuente de lo deseado y se viene convirtiendo en un serio problema de carácter global y una preocupación muy generalizada a nivel mundial (Álvarez y López, 2017; Tuero, Cervero, Esteban y Bernardo, 2018).

Y, si concretamos un punto más, tema de nuestro trabajo, el abandono que se produce en los estudios a distancia y soportado en sistemas digitales suele ser de forma más aguda un lugar común de debate permanente entre instituciones y expertos preocupados por el tema, dado que es superior al correspondiente en las instituciones presenciales (Carr, 2000; Moore, Bartkovich, Fetzner e Ison, 2003; Barefoot, 2004; Lee y Choi, 2011; Simpson, 2012; Waugh y Su-Searle, 2014). En efecto, la educación a distancia, en línea, los formatos educativos no presenciales, suelen sufrir de forma más aguda este problema que hoy se identifica como singular en el marco de las investigaciones internacionales (Hart, 2012; Álvarez y López, 2017).

En todo caso, esos diálogos rotos o deficientes que llevan al abandono, se convierten en un fracaso y, a veces, en un drama cuando las deserciones son elevadas, porque además de implicar al propio estudiante y a su entorno, afecta a todos los agentes sociales que pueden influir en el sistema universitario (Moncada, 2014), supone el fracaso de los docentes y, sin ninguna duda, el revés en su reputación de la propia institución (Willging y Johnson 2004; Liu, Gomez y Cherng, 2009) y del propio sistema (Grau y Minguillón, 2014; OCDE, 2012), con todo lo que ello supone, además, en términos socioeconómicos (Díaz, 2008; Colás, 2015; Vallejos y Steel, 2017). 
A pesar de lo que pudiera deducirse tras la lectura de este trabajo creemos, con Álvarez y López (2017), que quizás no se desarrolló suficiente investigación sobre este problema o quizás, más acertadamente, no se implementaron medidas concordantes con las conclusiones obtenidas en la investigación que sí se llevó a cabo, y se vino conviviendo con el problema considerándolo, quizás, como irresoluble, sin pensar que las acciones humanas siempre son susceptibles de mejora. O quizás, no se quiso ver o apreciar dicho problema, y vaya que es de grandes dimensiones (Woodley y Simpson, 2014).

Por tanto, en este trabajo nos planteamos dos objetivos principales, dado el componente social y relacional del fenómeno educativo (Vygotsky, 1979), por una parte, seleccionar aquellas causas que la investigación más destacada ha venido mostrando de forma reiterada, como debilitadoras del diálogo y, en consecuencia, como propiciadoras del abandono y, por otra, igualmente, con el apoyo de las investigaciones realizadas, apuntar una batería de acciones orientadoras y de apoyo, de carácter institucional y docente que pudieran promover diálogos pedagógicos de calidad que lleven al éxito, a mayores tasas de satisfacción (Mashburn, 2001), de rendimiento y, en consecuencia, de retención, persistencia y compromiso en los estudios (Canales y De los Ríos, 2018).

\section{LA METODOLOGíA DE ANÁLISIS}

El hilo conductor del trabajo está focalizado por los objetivos apuntados más arriba, causas del abandono y acciones para su reducción. Con el fin de confirmar que, respecto a las causas del abandono, éstas no cambiaron demasiado a lo largo de los años, salvo en lo que hace referencia a los aportes tecnológicos. De ahí que el origen sea un trabajo nuestro, de 1987, (García Aretio, 1987). A partir de ahí, utilizamos la herramienta "Linceo+" de la Biblioteca de la UNED de España. Se trata de un potente software que facilita, desde un único punto de consulta, la búsqueda y el acceso directo (en el caso de los recursos en línea) a la colección documental propia y de bases de datos, las más relevantes, con las que cuenta la Biblioteca UNED. De este modo el usuario se ahorra la necesidad de realizar sucesivas búsquedas de forma separada en distintos portales, interfaces y bases de datos.

Los criterios de búsqueda avanzada que seleccionamos en Linceo+, fueron: a) artículos publicados en revistas científicas; b) área de educación; c) seleccionar por términos en el título del artículo, y d) sólo artículos a partir del año 2000, y preferentemente referidos a estudios universitarios. Centramos la búsqueda en los siguientes términos: a) abandono, deserción, retención, persistencia y éxito, y b) relacionados con educación (enseñanza, aprendizaje) a distancia (virtual, en línea). Y todos los términos de búsqueda, realizados tanto en español como en inglés.

Los documentos que recogían sólo opiniones o datos no contrastados fueron eliminados, así como aquellos no publicados en revistas o editoriales reconocidos. Pusimos interés especial en detenernos sólo en trabajos que indagaban sobre las 
causas del abandono, y en su caso sobre el éxito, y sobre las medidas, métodos y recursos para reducir o atacar aquellos factores negativos. Prescindimos de estudios reiterativos sobre el tema, sobre todo en lo referente a las causas, las cuales cambian poco, aunque pueda variar la jerarquía de cada una, de unas investigaciones a otras.

Cierto que ha surgido variada y abundante literatura científica relacionada con este tema de estudio en el sentido de aplicar determinada herramienta, software o micrometodología, con la intención de reducir las deserciones. Salvo cuando se trató de herramientas o metodologías con cierto recorrido, también prescindimos de estos trabajos. Y todo ello, siempre que pudiéramos relacionarlo con nuestro enfoque centrado en el diálogo pedagógico.

Con esos criterios se consultaron un total de 127 trabajos de entre los que se seleccionaron para un análisis más detenido, 85 artículos, todos ellos citados en la bibliografía (referencias) de este trabajo, distribuidos así: abandono + dropout $=40$; deserción + attrition $=12$; retención + retention + persistencia + persistence $=27$; éxito + success $=6$.

\section{LOS RESULTADOS. LAS CAUSAS}

Parece evidente que el problema del abandono de los estudiantes que siguen cursos a través de formatos no presenciales, no presenta perfiles bien definidos y concretos, que pudieran abordarse de forma determinante, más bien al contrario, se trata de un fenómeno de naturaleza multidimensional, multifactorial, altamente complejo y en constante evolución (Carr, 2000; Pierrakeas, Xeno, Panagiotakopoulos y Vergidis, 2004; Holder, 2007; Bethencourt, Cabrera, Hernández, Álvarez y González, 2008; Celis, Flores, Reyes y Venegas, 2013; Juajibioy, 2016), más allá de que las causas más destacadas que lo generan vengan reiterándose en sucesivas investigaciones. Pero interesa refrescar esas causas, con el fin de que los profesionales de la educación que laboran en ambientes virtuales, puedan aplicar diseños y metodologías con la finalidad de reconducir esos diálogos rotos y reducir, así, esas tasas de deserción (Lim, 2016).

En general los modelos que vienen tratando de predecir o explicar la deserción de los estudiantes universitarios, con diferentes matices, vienen clasificando los numerosos indicadores de fracaso en torno, sobre todo, a estas categorías: estudiante, institución, docentes, medios, grado de integración social y académica, etc. (Pineda, Ortiz y Moreno, 2001; Tresman, 2002; Xenos, Pierrakeas y Pintelas, 2002; Rovai, 2003; Ashby, 2004; Cabrera, Tomás, Álvarez y Gonzalez, 2006; Levy, 2007; Vásquez y Rodríguez, 2007; Tello, 2007; Bethencourt y otros, 2008; Heyman, 2010; Street, 2010; Moon-Heum, 2012; Simpson, 2012; Koper, 2014; Cervero, Bernardo, Esteban, Tuero, Carbajal y Núñez, 2017; Himmel, 2018; Lott, Freitas, Ferreira y Lott, 2018).

Vamos a destacar algunas propuestas. Quizás, el recurrente modelo de Tinto (1975) que sirvió de base a estudios posteriores. De ese modelo nos fijamos sobre todo en su insistencia en que las percepciones de los estudiantes sobre su integración 
social y académica son influencias predominantes en la decisión de abandonar los estudios, o persistir en ellos. También deseamos destacar la amplia revisión de la literatura sobre el tema de Lee y Choi (2011), en la que abarcaron estudios de los últimos diez años, detectaron hasta 69 factores influyentes en la decisión de abandonar y los agruparon en tres grandes categorías: factores del estudiante, factores del curso o programa, y factores ambientales. Igualmente, Jancey y Burns (2013), distinguen entre factores referidos a la disposición del estudiante (motivación, confianza, actitud, aptitud, etc); factores de carácter socioeconómico y, finalmente, los de carácter institucional, en los que engloba la vertiente académica y docente. Y Jun (2005) estructura las variables de abandono en cinco categorías: antecedentes individuales, motivación, integración académica, integración social y entorno tecnológico.

Por su parte, Díaz (2008), tras encontrar seis predictores de la deserción estudiantil, propone un modelo para el abordaje del problema, muy similar al que nosotros manejamos para explicar diseños, procesos y valoraciones de las propuestas educativas a distancia y en línea, el del Diálogo Didáctico Mediado (DDM) (García Aretio, 2012 y 2014). Díaz (2008), partiendo de la literatura revisada, estructura su propuesta en tres ejes o grupos de factores que tratan de mantener cierto equilibrio: académico, institucional y social. Y dado que, en cualquier modelo pedagógico el centro es el estudiante habrán de considerarse también sus perfiles y características personales.

De esta manera, tras la consulta de tan diferentes modelos de análisis, como vemos en la figura 1, centramos nuestro estudio en el DDM, entendiendo que es el estudiante, con sus características, intereses, motivaciones, actitudes y aptitudes, el destinatario y protagonista de todo el quehacer en cualquier institución educativa. La dimensión social del diálogo implica que es el alumno el receptor principal del mismo, pero también, en muchas ocasiones, el emisor. 


\section{VARIABLES DE DESERCIÓN BASADAS EN EL DDM}

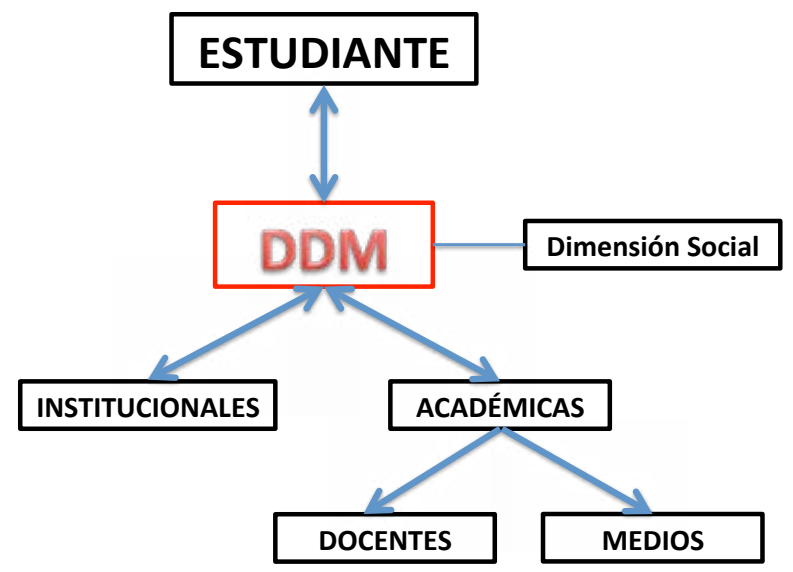

DDM: Diálogo Didáctico Mediado (García Aretio)

Parece, por tanto, importante indagar sobre la motivación de los estudiantes y sus características personales, sociales y demográficas, dado que ese conocimiento puede resultar de sumo interés para predecir el éxito y el fracaso (Jun, 2005; Simpson, 2013) y, por el contrario, cuáles otras o qué déficits de las primeras predisponen al fracaso. Deberían considerarse respecto de los estudiantes: su situación laboral, el compromiso inicial con la carrera elegida y con las metas parciales esperadas, las técnicas y habilidades de estudio poseídas, así como la calidad de salud y la relación con sus pares (Pigliapoco y Bogliolo, 2008; Tello, 2007). Aunque algunas de esas variables no puedan ser alteradas desde la perspectiva docente e institucional.

Morgado, Neves y Teixeira (2016), nos ofrecen un perfil ideal de estudiante en línea con una serie de características y competencias que, cuando no se muestran, suelen aparecer como factores influyentes en la decisión final de abandonar, tales como: competencias de comunicación escrita; espíritu abierto; responsabilidad y capacidad para la automotivación y autodisciplina; voluntad para estar dispuesto a compartir sus problemas y dificultades académicos; conciencia del grado de dificultad del curso; flexibilidad para aceptar argumentos y críticas de sus pares y profesores; aceptación del rol de un docente en línea más como facilitador que como transmisor de conocimiento; acceso a internet con competencias informáticas básicas; apertura al mundo digital; capacidad para la búsqueda, procesamiento y 
distribución de contenidos digitales; ética digital. En este caso, la mayor parte de esas variables, sí que podrían ser alteradas desde la perspectiva educativa.

Basándose en Simpson (2012), Sánchez-Elvira (2016), señala cuatro áreas de interés referidas a habilidades destacadas para que un estudiante a distancia pueda desenvolverse con cierta garantía: a) cognitivas, referidas fundamentalmente a las estrategias de aprendizaje; b) emocionales, control de la ansiedad y el estrés, la frustración, la soledad, etc.; c) organizativas, organización y autogestión del tiempo, y d) digitales, propias de la sociedad actual.

Si se les pregunta a los estudiantes, éstos suelen apuntar como principal causa de ruptura, la falta de tiempo para dedicar al estudio (Tresman, 2002; Street, 2010; Simpson, 2012 y 2013; Moncada, 2014). Si esos estudiantes no logran asentar hábitos de estudio sólidos tenderán a relajarse y, finalmente, abandonar, muy por encima de los que muestran buenos hábitos de trabajo intelectual (Johnston, 2002). Por otra parte, esa percepción de falta de tiempo pudiera deberse realmente a una organización institucional y docente deficiente respecto a los programas y cursos o de la propia orientación al estudiante para ayudarle en esa gestión y, quizás también a la tendencia a dejar el estudio para tiempo poco antes de los exámenes (procrastinación) (Wolters, 2003; González y Sánchez-Elvira, 2013). También puede considerarse, ante esa falta de tiempo, el problema de la frustración del estudiante en línea, ante el cúmulo de dificultades, sobre todo si es nuevo en estos escenarios, y que puede llevar al abandono (Borges, 2005; McGhie, 2017).

Esa falta de tiempo puede estar ligada a razones laborales, al tratar de compaginar el estudio con un determinado trabajo que puede restar horas y preocupación (García y Adrogué, 2015; Rué, 2014; Aguilera y Jiménez, 2012). Las obligaciones laborales de los estudiantes a distancia exigen a estos una adecuada gestión del tiempo, habilidad que se muestra como favorable a la persistencia en los estudios (Holder, 2007; Osborn, 2001; Sánchez y Elías, 2017). Mala opción la de no considerar realísticamente el tiempo del que van a disponer para el estudio y que deberán sustraer de otras actividades y obligaciones, subestimando la dedicación requerida para equilibrar sus obligaciones académicas y profesionales (Xenos y otros, 2002; Castles 2004; Pierrakeas y otros, 2004; Müller, 2008). De ahí la relevancia del aprendizaje autorregulado en los sistemas digitales de enseñanza y aprendizaje (Bol y Garner, 2011; González y Sánchez-Elvira, 2013; Cerezo, Bernardo, Esteban, Sánchez y Tuero, 2015). Las estrategias cognitivas y la regulación de la cognición son importantes para un aprendizaje en línea exitoso (Barak, Hussein-Farraj y Dori, 2016).

Tabbodi, Rahgozar y Makki (2015) señalan que el alumno abandona cuando rinde poco, y permanece cuando el rendimiento es positivo. La fórmula rendimientoresultados, parece determinante al adoptar o no la decisión final de desertar (DupinBryant, 2004; Moncada, 2014; Gairín, Triadó, Feixas, Figuera, Aparicio y Torrado, 2014; Choi y Park, 2018). 
Otras causas que destacan los investigadores: problemas tecnológicos (Frankola, 2001; Dupin-Bryant, 2004); las rigideces curriculares, la elección errónea de la carrera o acceder con expectativas equivocadas (Grebennikov y Shah, 2012); motivos económicos y sociales y la dificultad de los estudios (Duque, Duque y Surinach, 2013); la baja autoestima del estudiante (Roso-Bas, Pades y García, 2016); los bajos niveles de supervisión y apoyo docente (Montalvo, 2012); la escasa motivación, inadecuados diseños pedagógicos de las asignaturas y la baja cualificación de los docentes (Frankola, 2001); la escasa calidad de los programas con contenido relevante poco estructurados (Ivankova y Stick, 2007; Perry, Boman, Care, Edwards y Park, 2008); la falta de retroalimentación docente, la gran carga de trabajo y las dificultades para estudiar lejos del campus (Choi, Lee, Jung y Latchem, 2013). Por su parte, Fozdar, Kumar y Kannan (2006) ordenan las causas de abandono de esta manera: lejanía del centro de estudios, insuficiente apoyo académico, problema del tiempo de estudio, dificultad de asistir a los laboratorios, ausencia de interacción entre pares, dificultades para estudiar ciencias a distancia, dificultad del examen presencial, escasa capacidad de respuesta del centro de estudios.

Sánchez-Elvira (2016) llega a sintetizar una serie de causas, a) por una parte, de carácter institucional: centradas en la falta de información y orientación; materiales deficientes y complejos; problemas de comunicación-interacción; apoyo institucional débil, y, b) por otra, de carácter personal: formación previa insuficiente; déficit en competencias digitales; gestión del tiempo frente a la dificultad y a otras obligaciones; escasa motivación intrínseca, ansiedad académica, expectativas poco realistas, etc.; percepción de falta de competencia; carencia de estrategias y hábitos de estudio eficientes, en general, y especialmente aplicados a entornos en línea, en particular.

Existe un amplio estudio nuestro (García Aretio, 1987), de fecha muy lejana y, por tanto, en la que los apoyos tecnológicos eran los que eran y en el que tratamos de averiguar, por una parte, el perfil, habilidades y hábitos del estudiante universitario a distancia y su influencia en el rendimiento académico. Y por otra, acercarnos a las causas más ordinarias que llevan a los estudiantes a abandonar sus estudios.

Podemos afirmar que aquellas causas extraídas hace más de treinta años, sobre los estudiantes de la UNED de España, no resultan muy ajenas a las que hemos extraído tras nuestra revisión bibliográfica, salvedad hecha de los aportes digitales de hoy. Con respecto a las que podían hacer más referencia a los estudiantes, las causas, de mayor a menor incidencia, entonces eran éstas: atención a obligaciones laborales y familiares; falta de dedicación, de técnicas y hábitos de estudio; falta de motivación; falsas expectativas sobre los estudios a distancia; inasistencia a las sesiones tutoriales; deficiente formación básica, y escasos recursos económicos para afrontar gastos. En otro orden de cosas, otras causas, más ligadas a la responsabilidad de la institución y de los docentes, fueron entonces: complejidad y dificultad de las pruebas presenciales; ausencia de servicios de orientación; lejanía del centro asociado; dificultad y amplitud de los materiales de estudio; información 
escasa sobre la oferta de estudios y su adecuación a los intereses; excesiva y costosa bibliografía; inadecuación de los materiales para ser estudiados de forma autónoma; escasa ayuda recibida por parte de los docentes; sensación de ser tratado como un número; y deficiente preparación de los docentes. Otras de las señaladas en el área de responsabilidad del estudiante, igualmente podrían ser asignadas a la institución y docentes.

Tras la bibliografía consultada y las jerarquías de causas que algunos autores establecieron, estamos en condiciones de reelaborar este apartado, sugiriendo las razones, causas o factores que inclinan a los estudiantes a tomar tan drástica decisión, abandonar los estudios a distancia. Las organizaremos, tal y como habíamos señalado, en función de las variables contempladas en nuestra propuesta del DDM.

\section{Las causas atribuibles al estudiante}

Ha de destacarse que la influencia de las variables relativas al estudiante en la toma de decisiones respecto a su permanencia son las más importantes (Esteban, Bernardo, Tuero, Cerezo y Núñez, 2016). Desde la responsabilidad y el compromiso del adulto que decide inscribirse, matricularse, en una institución o programa a distancia, la literatura científica en general y nuestra revisión en particular, nos muestran este elenco de razones, causas o factores que podríamos calificar como más destacadas para romper la relación dialógica con los docentes e institución:

- La falta de tiempo. Generalmente se achaca a las obligaciones familiares (hijos, mayores a cargo, algún miembro dependiente, etc.) y a las laborales (horas de trabajo, cambio de responsabilidades laborales, cambio de trabajo, etc.). No se calculó bien el necesario equilibrio entre la necesaria dedicación al estudio y las demás obligaciones. O no se tuvo capacidad para planificar y organizar bien los tiempos y autorregular el aprendizaje.

- Deficientes técnicas de estudio. Carencia de habilidades, técnicas, estrategias y hábitos de estudio eficientes. Escasa capacidad para la concentración.

- Insuficiente motivación. Quizás nunca existió auténtico compromiso personal con los estudios que se iniciaron. Sin motivación intrínseca inicial resulta complicado ese compromiso. Sin motivación intrínseca de mantenimiento, que impulse la dedicación continuada y regulada al estudio, la ruptura está anunciada.

- Elección equivocada. No se contó con información suficiente ni con orientación personalizada a la hora de valorar qué tipo de estudios se van a iniciar. Las expectativas fueron poco realistas.

- Déficit de formación básica. Insuficiente para acometer un determinado tipo de estudios.

- Rendimiento académico bajo. Pobres rendimientos anteriores al acceso a la universidad, o bajos resultados iniciales o calificaciones negativas, provocan el desánimo. 
- Déficit en competencias digitales. Los problemas tecnológicos, bien sean por falta de conocimientos previos o por déficits de la propia institución, generan cortes comunicativos esenciales en cualquier proceso educativo.

- Escasa capacidad para la autonomía y la autodisciplina. Lo que provoca retrasar la decisión de acometer la tarea de estudiar con persistencia y rigor (procrastinar).

- Temor al fracaso. Lo que provoca ansiedad académica, agobio ante las dificultades y ante las distintas responsabilidades.

- Pobre integración académica y social. Una interacción deficiente con todos los agentes del proceso aproximará la sensación de que el estudiante se siente como un número, como algo impersonal. Sería el sentimiento de soledad y lejanía tan señalado en los trabajos anteriores a la era digital.

- Escasez de recursos económicos. A veces los gastos que provoca el seguimiento de un curso o carrera, sean costes de inscripción, sean de los materiales de estudio o de los componentes informáticos necesarios, pueden empujar a la deserción.

\section{Las causas que pueden atribuirse a los docentes}

- Escaso seguimiento y supervisión docente. Los profesores muestran bajos niveles de supervisión y apoyo al estudiante. La orientación académica es débil o inexistente, sea en los planos de carácter personal, académico o tecnológico.

- Los materiales de estudio carecen de un diseño adecuado. Existen carencias en el diseño instructivo o en la presentación de los recursos y materiales didácticos. O éstos son insuficientes o, por el contrario, demasiado extensos o complejos.

- Las rigideces curriculares. La falta de flexibilidad propia de los sistemas a distancia se ve mutilada por unas estructuras curriculares excesivamente pautadas y severas. El exceso de tarea y la descoordinación entre las diferentes asignaturas del curso, también genera desconexión.

- Pruebas de evaluación poco adecuadas. Sean las pruebas presenciales, si existen, o las pruebas de evaluación continua, sean éstas de autoevaluación, heteroevaluación o coevaluación, pueden ser desencadenantes de frustración.

- Baja cualificación de los docentes. Deficiente preparación de los instructores, más allá de su competencia académica, en lo que respecta a la capacitación en habilidades propias de los sistemas digitales de enseñanza y aprendizaje.

\section{Atribuibles a la institución}

- Deficiente información inicial sobre la carrera o curso.

- Ambigüedad en las directrices y orientaciones procedentes de la institución.

- Inexistencia de servicios de orientación. O servicios de orientación deficientes.

- Insuficiencia o inadecuación de los servicios tecnológicos. 


\section{RESPUESTAS AL ABANDONO DESDE EL DDM}

Si se detectan los problemas, causas o factores que generan la deserción o el éxito de los alumnos, será más fácil organizar planes de acción que puedan reducir ese problema (Huebner, 2013; Cambruzzi, Rigo y Barbosa, 2015). Las respuestas al abandono han de venir de la capacidad de docentes e institución de articular una relación dialógica y mediada de calidad, fluida y flexible con los estudiantes (DDM) que persiga neutralizar esas causas de deserción, tanto en la prevención de abandono temprano como en el subsiguiente (Gury, 2011). La relación de causas anotadas, supondría, en sí, suficiente programa de acción institucional y docente. En todo caso, la figura 2 esquematiza lo que deseamos transmitir, una institución y unos docentes que a través del DDM llevan al estudiante a la retención y persistencia y a la culminación de los estudios con éxito. ¿Cuáles deberán ser las preocupaciones institucionales y docentes para dar respuesta al problema del abandono? Consideraremos sólo aquellas que pueden afectar a variables alterables, es decir, obviaremos aquellas causas del abandono debidas a factores socioeconómicos de la situación personal, familiar y contextual del estudiante, que consideramos que no son alterables ni desde la perspectiva institucional ni docente. Pues bien, aproximémonos a las respuestas institucionales y docentes.

Figura 2. El DDM enfocado al éxito

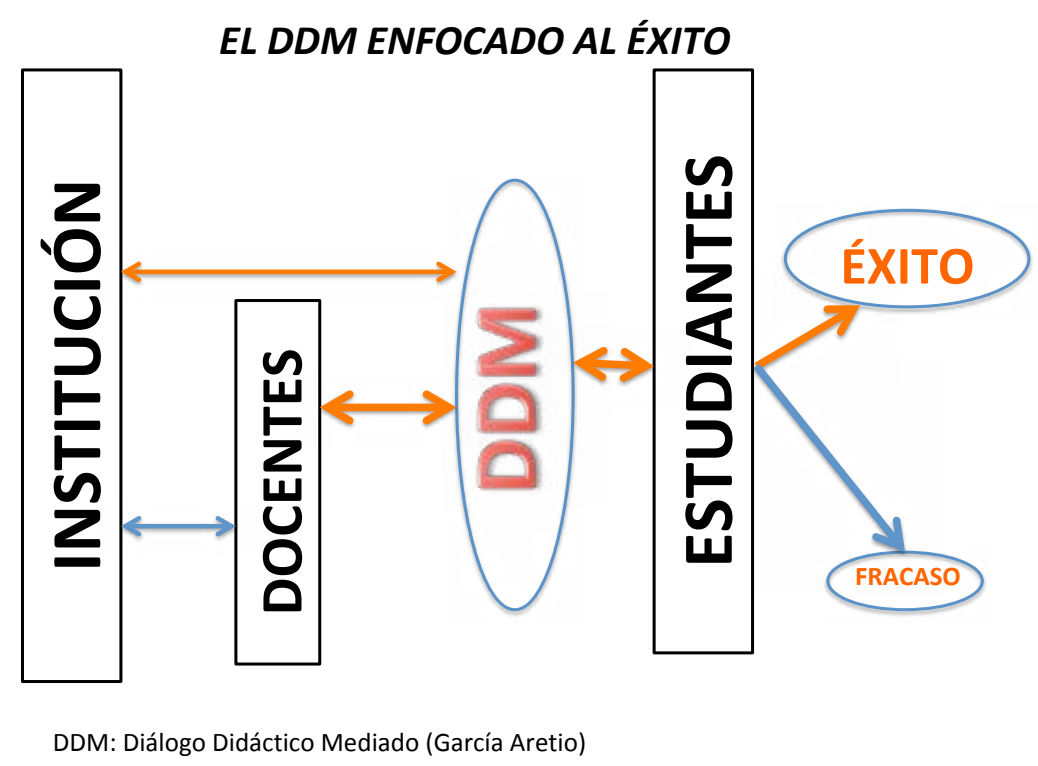




\section{Las respuestas desde la institución}

\section{a. Respecto a la falta de tiempo, las técnicas de estudio.}

La orientación haciala mejor gestión y organización del tiempo, la automotivación, el estudio autodirigido y autorregulado, se muestran como propuestas eficaces (Sun, 2014). Mientras más tiempo hayan dedicado al estudio, existen menores posibilidades de abandono (Park, Perry y Edwards, 2011). Por eso, la orientación (Borges, 2005) hacia la mejor gestión y organización del tiempo y el estudio autorregulado (Cerezo, Bernardo, Esteban, Sánchez y Tuero, 2015), se muestran como propuestas eficaces y resulta de importancia crítica para las modalidades a distancia (Nikolaki, Koutsouba, Lykesas, Venetsanou y Savidou, 2017). Autorregular el aprendizaje supone activar competencias clave como establecer metas, orientar la acción en consonancia con las metas, planificar, persistir, pedir auxilio cuando sea preciso, probar otras alternativas y reflexionar (Wang, 2010).

\section{b. La motivación.}

Al tratarse de un modelo pedagógico donde la responsabilidad recae básicamente en el estudiante, la falta de compromiso y dedicación (Vásquez y Rodríguez, 2007), ligado a la falta de motivación (Mahmodi y Ebrahimzade, 2015) ha venido siendo clave, como vimos, en los abandonos estudiantiles (Chyung 2001; Osborn 2001; Parker 2003; Levy, 2007; Ivankova y Stick 2007; Díaz, 2008; Park y Choi, 2009; Simpson, 2012), configurándose, en sentido contrario, en un gran predictor de permanencia. La implicación de toda la institución motivando y apoyando individualmente cuando existen problemas, se muestra como buena práctica que reduce el abandono (Gregori, Martínez y Moyano-Fernández, 2018). Si los beneficios de permanecer son percibidos como más elevados que los costos personales, entonces la permanencia tiene más posibilidades de éxito (Donoso y Schiefelbein, 2007).

Quizás, como apunta Simpson (2013), ha sobrado preocupación por facilitar unos materiales adecuados a los estudiantes, pero quizás faltó preocupación similar por una motivación inicial y de mantenimiento para el aprendizaje. En todo caso, que los estudiantes cuenten con las habilidades mínimas para enfrentarse a estudios en línea y dispongan de materiales apropiados y de calidad con los que sea fácil interaccionar (Zimmerman, 2012), así como que los docentes con el apoyo y recursos necesarios para enseñar, son propuestas que promueven la retención y el éxito (Roblyer, 2006).

\section{c. Escasa información y orientación inicial.}

Se hace preciso potenciar los servicios de orientación de estas instituciones (Frydeberg, 2007; Clay, Rowland y Packard, 2009). Orientar inicialmente hacia 
la carrera y el número de asignaturas a matricularse. Para ello, podrían activarse: guías generales, claras, y con información dirigida al que no conoce los sistemas a distancia; otros documentos de texto, audio y vídeo, motivadores, intuitivos y claros, que ayuden al estudiante a medir sus posibilidades, sus capacidades y tiempo previsto de dedicación al estudio. Muchos alumnos perciben que en estas universidades a distancia sería sencillo aprobar (García y Adrogué, 2015; Bernardo, Cervero, Esteban, Fernández y Núñez, 2016). Otros suelen subestimar las necesidades de tiempo para dedicarlo al estudio, manteniendo expectativas erróneas sobre sus posibilidades de permanencia (Pierrakeas y otros, 2004; Müller 2008). Se hace preciso orientarles porque así ajustan de forma realista el número de asignaturas matriculadas con la previsión de disposición de tiempo de estudio, experiencia y competencias previas (Castls, 2004; Dupin-Bryant, 2004; Holder, 2007; Rovai, 2003).

Liu y otros (2009), proponen unos cuestionarios automatizados de autoevaluación para orientar al estudiante aspirante, mostrando sus niveles de competencias ya poseídas, la disponibilidad de tiempo para el estudio (compromiso), la motivación hacia determinada carrera o profesión, etc. Tampoco deben olvidarse, sobre todo antes de la matrícula y en los primeros meses, unos servicios de apoyo administrativo eficaces (Muilenburg y Berge, 2001).

\section{d. El abandono temprano. La integración académica y social. Cursos cero y de acogida.}

En buena parte de los estudios citados se constata que el abandono se produce fundamentalmente a lo largo del primer año académico (Corominas, 2001). Siempre ha supuesto un serio hándicap el tránsito desde los estudios secundarios a la universidad (Álvarez, Figuera y Torrado, 2011; Álvarez y López, 2017; McGhie, 2017; Tuero y otros, 2018). Y si ese tránsito se realiza a una institución a distancia, la confrontación puede ser mayor. Y, además, en estas universidades vienen a ingresar todos los aspirantes, también aquellos estudiantes con expedientes académicos muy pobres, rozando el mínimo de lo preceptivo. Y ya está más que probado el hecho de que rendimientos anteriores predicen bien los éxitos o fracasos posteriores.

De ahí la relevancia de los cursos cero, cursos que, por su contenido de carácter básico, permiten al alumno nivelar sus conocimientos con los mínimos requeridos para iniciar el estudio de las asignaturas de la titulación. Generalmente son abiertos, sin fecha de inicio y fin y no precisan de inscripción previa (Cerezo, Núñez, Fernández, Suárez y Tuero, 2011; Rodríguez-Muñiz y Díaz, 2015; Tuero y otros, 2018). Igualmente, se deberían programar acciones que lleven al nuevo estudiante a entender como casi necesaria la realización de los cursos de acogida o de integración en la institución (Álvarez y López, 2017; Crawford y Persaud, 2013), atendiendo a: orientar sus primeros pasos en la universidad; mostrarles las características de la institución, sus metodologías y apoyos a su alcance; brindar posibilidades iniciales para la adquisición de aquellas competencias tecnológicas no poseídas y necesarias 
para estudiar en una universidad digital; adentrarles en la plataforma virtual oficial; ayudar a reforzar el compromiso del estudiante en la decisión de matricularse en una carrera, estudiar, cumplir tareas, etc.

Ya sabemos que reducen la frustración los esfuerzos de socialización e integración de los estudiantes (Conrad, 2002). El trabajo colaborativo suele aumentar el sentido de pertenencia al grupo y así prevenir la desmotivación (Lee, 2000), dado que, superado el primer año, el nivel de motivación cambia, al relacionarse con la integración académica y social (Díaz, 2008). También resulta positiva la celebración de reuniones virtuales en pequeños grupos de alumnos (Moody, 2004).

Se haría preciso identificar a los estudiantes en riesgo y proporcionarles oportunidades para recuperar el diálogo (Dupin-Bryant, 2004; Perry y otros, 2008). Así, ejercer alguna atención específica a aquellos alumnos que no cumplen plazos en entregas de las primeras actividades, tareas o trabajos obligatorios, tales como establecer un protocolo de mensajes o llamadas a esos posibles abandonos, que vienen produciendo efectos positivos (Burgos, Campanario, Peña, Lara, Lizcano y Martínez, 2018).

\section{e. La apuesta tecnológica}

Se haría necesario potenciar todos los servicios tecnológicos de apoyo a una docencia de calidad con la que deben contar estas instituciones. La actividad educativa soportada en sistemas digitales genera una ingente cantidad de datos, rastros de navegación y actividad, que tratados adecuadamente a través de minerías o analíticas pueden ofrecernos pistas fiables para actuaciones futuras dirigidas a reforzar acciones que propicien el buen rendimiento y la satisfacción del estudiante (Romero y Ventura, 2010). Parece que la aplicación rigurosa de analíticas de aprendizaje para detectar predictores de éxito y de abandono en cursos en línea de determinados perfiles y actitudes estudiantiles, resulta cada vez más eficaz (Yasmin, 2013). Finalmente, quizás se hace preciso un énfasis en la formación previa en esta competencia al estudiante en línea (Borges, 2005), sin olvidar a los docentes, a los que ha de atenderse y mantener permanentemente actualizados (Holmgren y Johansson, 2012).

\section{Las respuestas desde la docencia}

Gaytan (2015) refuerza la afirmación de que a los estudiantes en línea les agrada recibir instrucciones de sus profesores y comentarios lo más completos posibles que les permitan involucrarse en el proceso para mejorar el rendimiento. Desde este enfoque, dentro de las competencias y compromisos de carácter pedagógico, podríamos destacar como buenas prácticas que habría de desarrollar el docente (o los equipos docentes) enfocadas al logro de diálogos que generen aprendizajes de calidad, a la satisfacción del estudiante (Mashburn, 2001; Moore y otros, 2003; Levy 
2007; Donoso y Schiefelbein, 2007) y al desarrollo de competencias que reduzcan el abandono, las siguientes:

\section{a. Elaborar la Guía de estudio}

La Guía de estudio, Guía didáctica o Guía docente, es un elemento sustancial (García Aretio, 2001) del que nos valemos para orientar el estudio, acercando a los procesos cognitivos del alumno el material didáctico, con el fin de que pueda trabajarlo de manera autónoma. Es una pieza clave e indispensable (García Cabrera y otros, 2010) para el desarrollo del proceso de enseñanza a distancia (Aguilar, 2004) y que, por tanto, puede suponer la base del diseño de la asignatura o curso en cuestión. Y la relevancia del diseño en programas y cursos, está probada a efectos de la retención de los estudiantes (Tresman, 2002; Burns, 2013; Creelman y Reneland, 2013). Una guía realista, plausible, práctica y siempre actualizada, reducirá las demandas de apoyo por parte del estudiante, y se convertirá en un instrumento ideal para la autorregulación del aprendizaje (Cabaní y Carretero, 2003).

En esta guía habrían de contemplarse como mínimo: las competencias y resultados de aprendizaje; los objetivos de aprendizaje, claros, explícitos y evaluables; los contenidos apropiados para la consecución de objetivos y competencias; las actividades y trabajos individuales y colaborativos obligatorios y optativos; las prácticas obligatorias, en su caso; la evaluación formativa, continua y sumativa; las vías y formato de comunicación estudiantes-docentes; la metodología didáctica apropiada, y el plan de trabajo con la carga horaria prevista. Y todo ello, cuidando al máximo la consistencia y coherencia absoluta entre todas las variables curriculares contempladas en la Guía (García Aretio, 2001 y 2014).

\section{b. Diseñar, seleccionar y/o elaborar los diferentes materiales de estudio.}

Materiales adaptados al entorno virtual que da soporte a la asignatura o curso, tales como: textos impresos, sólo si procede; material digital principal o complementario; presentaciones fijas o animadas de los temas; minimódulos, minivídeos o videoclases; preparación de videoconferencias o webconferencias; elaboración de diferentes pruebas y formularios de evaluación; preguntas frecuentes (FAQ); glosario de términos o conceptos relevantes. Y ello, considerando la medida adecuada de los créditos o carga de trabajo del estudiante, así como la consistencia absoluta con lo propuesto en las guías. Sea cual fuere el formato de los materiales, estos deberían ser interactivos, flexibles y con posibilidades de autoestudio, con el fin de que los estudiantes accedan a ellos con la máxima facilidad y puedan entenderlos mejor (Ivankova y Stick, 2007; Müller, 2008; Perry y otros, 2008; García Aretio, 2014). En todo caso, es necesario revisar la estructura didáctica del material y los recursos (Tladi, 2013). 


\section{c. Gestionar la actividad docente.}

Ello conlleva: ajustarse prioritariamente a lo establecido en la Guía, que inicialmente es lo esperado por los estudiantes; propiciar oportunidades de aprendizaje tanto individual como grupal; aplicar diferentes estrategias metodológicas; orientar el aprendizaje adaptándolo al perfil del estudiante o grupo; integración académica y social de los estudiantes de nuevo ingreso, dado que la vinculación positiva entre estudiantes y profesores se revela como factor de retención en los estudios (Bernardo y otros, 2016; Castejón, Gilán, Miñano y Veas, 2016; Abello, Vila, Pérez, Lagos, Espinoza y Díaz, 2016; Tuero y otros, 2018); seleccionar, activar y gestionar las diferentes herramientas y aplicaciones digitales, internas o externas (web 2.0) a la plataforma; activar y gestionar la comunidad virtual, a través de la comunicación vertical y horizontal, síncrona y asíncrona, propiciando el diálogo didáctico mediado óptimo (García Aretio, 2014), facilitando la interacción de los estudiantes con el contenido (Zimmerman, 2012), con los profesores y con los otros estudiantes (Swan, 2001; Tello, 2007; Pigliapoco y Bogliolo, 2008; Ivankova y Stick, 2007), diálogo e interacción multifocal que es la que con mayor fuerza inclina a un estudiante a proseguir sus estudios o a abandonar (Holder, 2007; Perry y otros, 2008); celebración de videoconferencias o webconferencias; gestionar las diferentes actividades y tareas que han de realizar los alumnos. Y ello siempre, considerando la consistencia y coherencia absoluta entre las expectativas, lo esperado, lo diseñado, desarrollado y evaluado (García Aretio, 2014).

Ofrecer tutorías no solo reactivas (resolución de dudas) sino proactivas, anticipando problemas, lagunas de los materiales, etc. Se trata de orientar, facilitar, resolver dudas, dinamizar, motivar, etc., mediante metodologías activas y colaborativas. Simpson (2013) insiste en la relevancia del contacto proactivo para superar la deserción. La decidida presencia docente en el curso virtual fomenta la participación y aminora los sentimientos de soledad o distancia. También deberían valorarse las posibilidades de los sistemas de mentorías y acompañamiento o tutorías entre iguales (Fernández, Arco, López y Heilborn, 2011).

\section{d. Activar los diferentes modelos y formas de evaluar.}

En este aspecto, resulta de interés: cuidar las pruebas presenciales y su concordancia con lo estudiado; consolidar fórmulas de evaluación continua y formativa a través de las diferentes herramientas tecnológicas; valorar con efectos evaluadores las diferentes situaciones en las que un alumno en línea puede mostrar sus habilidades y competencias; explicitar con máxima claridad la tipología, características, criterios de valoración, duración, etc., de las pruebas presenciales, así como las diferentes ponderaciones de las restantes instancias evaluadoras; informar a los alumnos de forma sistemática, continuada y motivada de sus progresos. 
Proponer diferentes pruebas de autoevaluación (evaluación automática); facilitar pruebas de evaluación similares a las pruebas presenciales, con el objetivo de familiarizar al estudiante con las mismas; establecer criterios para la valoración del seguimiento de estudiantes a través de sus aportaciones en los foros, chats, trabajos colaborativos, etc.

\section{CONCLUSIÓN}

Nuestra propuesta de modelo está basada en el diálogo (DDM). En primer lugar, a través de su dimensión social y emocional, que hace referencia a las diferentes vertientes comunicativas del estudiante con los docentes, con la institución y con sus iguales (García Aretio, 2014). El diálogo se convierte en elemento generador de motivación, de ambientes propicios para el aprendizaje y de sentido de pertenencia y, por tanto, de reductor de los sentimientos de soledad y lejanía (Moore y otros, 2003). Por su parte, la dimensión pedagógica/académica entiende que ese diálogo ha de tender a la adquisición de conocimientos y habilidades, es decir, hacia una relación de carácter didáctico. Y, finalmente, el carácter o componente de "mediado" ha de considerar la dimensión de los recursos y la tecnología, siempre importante, pero en sistemas de enseñanza y aprendizaje digitales, imprescindible.

Y si ese diálogo no es de calidad, no es eficaz, sea por déficit del estudiante, o sea por rupturas con la institución (causas institucionales), con los docentes o con los recursos (causas académicas), o con los propios pares, la deserción podrá estar más cerca. Según el modelo que proponemos, el problema siempre será de carácter relacional, dialógico, conversacional, informacional y comunicacional, porque, promover el diálogo o no promoverlo acarrea consecuencias importantes en la selección de la información educativa relevante y su posterior conversión en conocimiento (Prieto y Duque, 2009). O bien el estudiante inició estudios donde no debía, o le faltaban actitudes o aptitudes, o todo a la vez; o bien la institución no tenía una oferta clara, bien definida, motivada y debidamente informada, o no puso las infraestructuras necesarias para ese diálogo fluido; o bien los docentes propiciaron la ruptura de esa relación, sea en el plano meramente académico, en el emocional, social, etc., que configurase un clima no propicio para el aprendizaje; o, finalmente, el diálogo con los medios y recursos tecnológicos resultaba costoso, complejo o confuso, fuese por un deficiente diseño de materiales o fuese por la dificultad intrínseca de determinada tecnología para unos destinatarios concretos (Montalvo, 2012). También podría contemplar déficits de diálogo la relación del estudiante con sus iguales.

En consecuencia, desde la institución y desde la actividad docente, han de implementarse medidas y acciones concretas para restablecer esos diálogos débiles o rotos y aumentar la retención (Lim, 2016), tales como: promover cursos de acogida para los nuevos alumnos; propiciar líneas para que los estudiantes aprendan a autorregular el estudio; motivar para la permanencia; sistematizar acciones 
informativas y de orientación hacia la elección de carrera; promover la integración académica y social, con énfasis en el primer año; potenciar los servicios tecnológicos de apoyo a la docencia y el aprendizaje; impulsar una docencia de calidad adaptada a los sistemas digitales de enseñanza y aprendizaje, con diseños rigurosos, gestión eficaz de la docencia en línea y activando diferentes formas y modelos de evaluación formativa.

\section{REFERENCIAS}

Abello, R., Vila, I., Pérez, M. V., Lagos, I., Espinoza, C., y Díaz, A. (2016). Identidad de aprendiz como herramienta analítica de experiencias universitarias en el contexto de la permanencia y el abandono: propuesta teórica. Paideia, 58, 11-34.

Aguilar R. (2004). La Guía Didáctica, un material educativo para promover el aprendizaje autónomo. Evaluación y mejoramiento de su calidad en la modalidad abierta y a distancia de la UTPL. RIED. Revista Iberoamericana de Educación a Distancia, 7(1-2), 179-192. doi: https://doi.org/10.5944/ried.7.1$\underline{2.1082}$

Aguilera, M. N., y Jiménez, V. E. (2012). Factores de deserción universitaria en el primer curso de las carreras de Trabajo Social y Lengua Inglesa en las Facultades de Humanidades y Ciencias de la Educación y de Lenguas Vivas de la Universidad Evangélica de Paraguay. Revista Internacional de Investigación en Ciencias Sociales, 8(2), 197-205.

Álvarez, M., Figuera, P., y Torrado, M. (2011). La problemática de la transición bachillerato-universidad en la Universidad de Barcelona. Revista Española de Orientación y Psicopedagogía, 22(1), 1527.

Álvarez, P. R., y López, D. (2017). Estudios sobre deserción académica y medidas orientadoras de prevención en la universidad de La Laguna (España). Revista Paradigma, 38(1), 48-71, Recuperado de https://goo.gl/mC5SM3.
Ashby, A. (2004). Monitoring Student Retention in the Open University: Detritions, measurement, interpretation and action. Open Learning, 19(1), 65-78.

Barak, M., Hussein-Farraj, R., y Dori, Y. J. (2016). On-campus or online: examining self-regulation and cognitive transfer skills in different learning settings. International Journal of Educational Technology in Higher Education, 13(1), 35. doi: https://doi.org/10.1186/s41239016-0035-9

Barefoot, B. O. (2004). Higher Education Revolving Door: Confronting the problem of student dropout in US colleges and University. Open Learning, 19(1), 9-18.

Bernardo, A., Cervero, A., Esteban, M., Fernández, E., y Núñez, J. C. (2016). Influencia de variables relacionales y de integración social en la decisión de abandonar los estudios en Educación Superior. Psicologia, Educação e Cultura, $X X(1), 138-151$.

Bethencourt, J., Cabrera, L., Hernández, J., Álvarez, P., y González, M. (2008). Variables psicológicas y educativas en el abandono universitario. Revista Electrónica de Investigación Psicoeducativa, 6(3), 603-622.

Bol, L., y Garner, J. K. (2011). Challenges in supporting self-regulation in distance education environments. Journal Computer High Education, 23, 104-123.

Borges, F. (2005). La frustración del estudiante en línea. Causas y acciones preventivas. Digithum. Revista electrónica de los Estudios de 
Humanidades y Filosofía de la UOC, 7. Recuperado de https://goo.gl/cuXgLC

Broc, M. A. (2011). Voluntad para estudiar, regulación del esfuerzo, gestión eficaz del tiempo y rendimiento académico en alumnos universitarios. Revista de Investigación Educativa, 29(1), 171-185.

Burgos, C., Campanario, M. L., Peña, D. d. l., Lara, J. A., Lizcano, D., y Martínez, M. A. (2018). Data mining for modeling students' performance: A tutoring action plan to prevent academic dropout. Computers and Electrical Engineering, 66, 541-556. doi: 10.1016/j.compeleceng.2017.03.005.

Burns, M. (2013). Staying or leaving? Designing for persistence in an online educatortraining programmein Indonesia. Open Learning: The Journal of Open, Distance and e-Learning, 28(2), 141-152. doi: 10.1080/02680513.2013.851023.

Cabaní, M. L. y Carretero, R. (2003). La promoción de estudiantes estratégicos a través del proceso de evaluación que proponen los profesores universitarios. En C. Monereo, C. y Pozo, J.I. La Universidad ante la nueva cultura educativa. Madrid: Síntesis.

Cabrera, L., Tomás, J., Álvarez, P., y González, M. (2006). El problema del abandono de los estudios universitarios. RELIEVE, 12(2). Recuperado de http://www.uv.es/ RELIEVE/v12n2/RELIEVEv12n2 1.htm

Cambruzzi, W., Rigo, S. J., y Barbosa, J. L. (2015). Dropout Prediction and Reduction in Distance Education Courses with the Learning Analytics Multitrail Approach. Journal of Universal Computer Science, 21(1), 23-47.

Canales, A., y De los Ríos, D. (2018). Factores explicativos de la deserción universitaria. Calidad en la Educación, o(26). doi: https://doi.org/10.31619/caledu.n26.239

Carr, S. (2000). As distance education comes of age, the challenge is keeping the student. Chronicle of Higher Education, 46 (23), 39-41.
Castejón, J., Gilán, R., Miñano, P., y Veas, A. (2016). Identificación y establecimiento de las características motivacionales y actitudinales de los estudiantes con rendimiento académico menor de lo esperado según su capacidad (underachievement). European Journal of Education and Psychology, 9(2), 63-71.

Castles, J. (2004). Persistence and the adult learner: Factors affecting persistence in Open University students. Active Learning in Higher Education, 5(2), 166179.

Celis, R., Flores, C., Reyes, M., y Venegas, H. (2013). Factores de riesgo de deserción presentes en alumnos repitentes de las carreras de Enfermería y Kinesiología en una universidad chilena. Ciencia $y$ enfermería, 19(3), 63-71. Recuperado de https://dx.doi.org/10.4067/So71795532013000300007

Cerezo, R., Bernardo, A., Esteban, E., Sánchez, M., y Tuero, E. (2015). Programas para la promoción de la autorregulación en educación superior: un estudio de la satisfacción diferencial entre metodología presencial y virtual. European Journal of Education and Psychology, 8(1), 30-36.

Cerezo, R., Núñez, J. C., Fernández, E., Suárez, N., y Tuero, E. (2011). Programas de intervención para la mejora de las competencias de aprendizaje autorregulado en educación superior. Perspectiva educacional, 5O(1), 1-30.

Cervero, A., Bernardo, A., Esteban, M., Tuero, E., Carbajal, R., y Núñez, J. (2017). Influencia en el abandono universitario de variables relacionales y sociales. Revista de Estudios e Investigación en Psicología y Educación, o(12), 46-49. doi: http:// dx.doi.org/10.17979/reipe.2017.0.12.2531

Chyung, S. (2001). Systematic and systemic approaches to reducing attrition rates in online higher education. American Journal of Distance Education, 15(3), 3649. 
Choi, H., Lee, Y., Jung, I., y Latchem, C. (2013). The extent of and reasons for non re-enrolment: A case of Korea National Open University. The International Review of Research in Open and Distributed Learning, 14(4). doi: http:// dx.doi.org/10.19173/irrodl.v14i4.1314

Choi, H., y Park, J. (2018). Testing a pathanalytic model of adult dropout in online degree programs. Computers \& Education, 116, 130-138.

Clay, M. N., Rowland, S., y Packard, A. (2009). Improving undergraduate online retention through gated advisement and redundant communication. Journal of College Student Retention: Research, Theory and Practice, 10(1), 93-102.

Colás, P. (2015). El abandono universitario. Revista Fuentes, 16, 9-14.

Conrad, D. L. (2002). Engagement, excitement, anxiety, and fear: learners experiences of starting an online course. The American Journal of Distance Education, 16(4), 205-226.

Corominas, E. (2001). La transición a los estudios universitarios. Abandono o cambio en el primer año de universidad. Revista de Investigación Educativa, 19(1), 127-151.

Crawford, C., y Persaud, C. (2013). Community Colleges Online. Journal of College Teaching \& Learning - First Quarter, 10(1), 75-86.

Creelman, A., y Reneland-Forsman, L. (2013). Completion rates. A false trail to measuring course quality? Let's call in the HEROEs instead. European Journal of Open, Distance and E-learning, 16(2), 40-49.

Díaz, C. (2008). Modelo conceptual para la deserción estudiantil universitaria chilena. Estudios pedagógicos, 34 (2), 65-86. Recuperado de https://goo.gl/ f2Y1RW

Donoso, S., y Schiefelbein, E. (2007). Análisis de los modelos explicativos de retención de estudiantes en la Universidad: Una visión desde la desigualdad social. Estudios Pedagógicos XXXIII, (7-27).

Duque, L. C., Duque, J. L., y Surinach, J. (2013). Learning outcomes and dropout intentions: an analytical model for Spanish universities. Educational Studies, 39(3), 261-284.

Dupin-Bryant, P. (2004). Pre-entry variables related to retention in online distance education. American Journal of Distance Education, 18(4), 199-206.

Esteban, M., Bernardo, A., Tuero, E., Cerezo, R., y Núñez, J. (2016). El contexto sí importa: identificación de relaciones entre el abandono de titulación y variables contextuales. European Journal of Education and Psychology, 9(2), 79-88.

Fernández, F., Arco, J. L., López, S., y Heilborn, V. A. (2011). Prevención del fracaso académico universitario mediante tutoría entre iguales. Revista Latinoamericana de Psicología, 43(1), 59-71.

Flecha, R., Gómez, J., y Puigvert, L. (2001). Teoría sociológica contemporánea. Barcelona: Paidós.

Fozdar, B., Kumar, L., y Kannan, S. (2006). Study of the Factors Responsible for the Dropouts from the BSc Programme of Indira Gandhi National Open University. The International Review of Research in Open and Distributed Learning, 7(3). doi: $\quad$ http://dx.doi.org/10.19173/irrodl. v7i3.291

Frankola, K. (2001). Why online learners dropout. Workforce Management, 80 , 53-58.

Frydenberg, J. (2007). Persistence in university continuing education online classes. International Review of Research in Open and Distance Learning, 8(3), 1-15.

Gairín, J., Triadó, X. M., Feixas, M., Figuera, P., Aparicio, P., y Torrado, M. (2014). Student dropout rates in Catalan universities: profile and motives 
for disengagement. Quality in Higher Education, 2O(2), 165-182.

García Aretio, L. (1987). Rendimiento académico y abandono en la educación superior a distancia. Madrid: UNED.

García Aretio, L. (1992). Hacia una teoría de la educación a distancia. En VV.AA. Cuestiones actuales sobre educación, 97118. Madrid: UNED.

García Aretio, L. (2001). La educación a distancia. De la teoría a la práctica. Barcelona: Ariel.

García Aretio, L. (2011). Perspectivas teóricas de la educación a distancia y virtual. Revista española de pedagogía, 249, 255-271.

García Aretio, L. (2014). Bases, mediaciones y futuro de la educación a distancia en la sociedad digital. Madrid: Síntesis.

García, A., y Adrogué, C. (2015). Abandono de los estudios universitarios: dimensión, factores asociados y desafíos de la política pública. Revista Fuentes, 16, 85106. doi: http://dx.doi.org/10.12795/ revistafuentes.2015.116.04

García, L., Ortega, J., Peña, M., Ruano, I., y Ortiz, A. (2010). La calidad en la docencia virtual: la importancia de la guía de studio. Pixel-Bit, 37, 77-92.

Gaytan, J. (2015) Comparing Faculty and Student Perceptions Regarding Factors That Affect Student Retention in Online Education, American Journal of Distance Education, 29(1), 56-66, doi: 10.1080/08923647.2015.994365.

González-Brignardello, M. P., y SánchezElvira-Paniagua, A. (2013). ¿Puede amortiguar el engagement los efectos nocivos de la procrastinación académica? Acción Psicológica, 10, 115-132. doi: http://dx.doi.org/10.5944/ap.10.1.7039

Grau, J., y Minguillón, J. (2014). Rethinking dropout in online higher education: The case of the Universitat Oberta de Catalunya. The International Review of Research in Open and Distributed
Learning, 15(1). doi: http://dx.doi. org/10.19173/irrodl.v15i1.1628

Gregori, P., Martínez, V., y MoyanoFernández, J. J. (2018). Basic actions to reduce dropout rates in distance learning. Evaluation and Program Planning, 66, 48-52. doi: 10.1016/j. evalprogplan.2017.10.004.

Grebennikov, L., y Shah, M. (2012). Investigating attrition trends in order to improve student retention. Quality Assurance in Education, 2O(3), 223-236.

Gury, N. (2011). Dropping out of higher education in France: a microeconomic approach using survival analysis. Education Economic, 19(1), 51-64. doi: 10.1080/09645290902796357.

Hart, C. (2012). Factors Associated With Student Persistence in an Online Program of Study: A Review of the Literature. Journal of Interactive Online Learning, 11(1), 9-42.

Heyman, E. (2010). Overcoming student retention issues in higher education online. Journal of Distance Education, 22, 72-89.

Holder, B. (2007). An investigation of hope, academics, environment, and motivation as predictors of persistence in higher education online programs. Internet and Higher Education, 10(4), 245-26o.

Holmgren, R., y Johansson, S. (2012). Reducing Dropouts in Online Education: Group Tutoring in Virtual Seminars and Support Structures. US-China Education Review A 11, 2(11), 971-978.

Huebner, R. A. (2013). A survey of Educational Data Mining research. Research in Higher education Journal, 19, 10-23.

Ivankova, N. V., y Stick, S. L. (2007). Students persistence in a distributed doctoral program in educational leadership in higher education: A mixed methods study. Research in Higher Education, 48(1), 93135 . 
Jancey, J., y Burns, S. (2013). Institutional factors and the postgraduate student experience. Quality Assurance in Education, 21(3), 311-322, doi: https:// doi.org/10.1108/QAE-Nov-2011-0069

Johnston, V. (2002). Improving Student retention-By accident or design. Exchange, 1.

Juajibioy, J. C. (2016). Study of University Dropout Reason Based on Survival Model. Open Journal of Statistics, 6, 908-916. doi: http://dx.doi.org/10.4236/ ojs.2016.65075

Jun, J. (2005). Understanding E-dropout? International Journal on E-Learning, 4(2), 229-240

Koper,E.J.R.(2014).Towardsamoreeffective model for distance education. E-Learning and Education, 10. Recuperado de http:// eleed.campussource.de/archive/10/4010

Lee, Y. (2000). Student motivation in the online learning environment. Journal of Educational Media \& Library Sciences, 37(4), 367-375. Recuperado de http:// coreylee.me/en/publications/2000 Student motivation.pdf

Lee, Y., y Choi, J. (2011). A review of online course dropout research: implications for practice and future research. Education Technology Research Dev, 59, 593-618. doi: 10.1007/s11423-010-9177-y.

Levy, Y. (2007). Comparing dropouts and persistence in e-learning courses. Computers \& Education 48, 185-204.

Lim, J. (2016). Predicting successful completion using student delay indicators in undergraduate self-paced online courses. Distance Education, 37(3), 317332.

Liu, S., Gomez, J., y Cherng, Y. (2009). Community college online course retention and final grade: Predictability of social presence. Journal of Interactive Online Learning, 8(2), 165-182.

Lott, A., Freitas, A., Ferreira, J., y Lott, Y. (2018). Persistência e Evasão na Educação a Distância: Examinando
Fatores Explicativos. Revista Eletrônica de Ciência Administrativa, 17(2), 149171. doi: https://doi.org/10.21529/ RECADM.2018006

Mahmodi, M., y Ebrahimzade, I. (2015). The analysis of Iranian students' persistence in online education. The International Review of Research in Open and Distributed Learning, 16(1). doi: http:// dx.doi.org/10.19173/irrodl.v16i1.1982

Mashburn, A. J. (2001). A psychological process of college student dropout. Journal of College Student Retention: Research, Theory and Practice, 2(3), 173190.

McGhie, V. (2017). Entering university studies: identifying enabling factors for a successful transition from school to university. Higher Education Journal, 73, 407-422.

Moncada Mora, L. (2014). La integración académica de los estudiantes universitarios como factor determinante del abandono de corto plazo. Un análisis en el Sistema de Educación Superior a Distancia del Ecuador. RIED. Revista Iberoamericana de Educación a Distancia, 17(2), 173-196. doi: https:// doi.org/10.5944/ried.17.2.12683

Montalvo, D. R. (2012). La retención y la deserción en línea: fenómeno de un modelo educativo virtual. HETS Online Journal, 2(2).

Moody, J. (2004). Distance Education. Why are the Attrition Rates so High? Quarterly Review of Distance Education, 5(3), 205210.

Moon-Heum, Ch. (2012). Online student orientation in higher education: a developmental study. Education Technology Research Dev, 6o, 1051-1069.

Moore, K., Bartkovich, J., Fetzner, M., e Ison, S. (2003). Success in cyberspace: Student retention in online courses. Journal of Applied Research in the Community College, 1O(2), 107-118. 
Morgado, N., Neves, A., y Teixeira, A. (2016). Acolhimento e integração como valor estratégico: análise do sistema institucional de apoio ao estudante virtual da UAb. En M. Cruz y A. Sánchez-Elvira (Eds.), Claves innovadoras para la prevención del abandono en instituciones de educación abierta y a distancia (2756). Santiago de los Caballeros: UAPAAIESAD.

Muilenburg, L., y Berge, Z. L. (2001). Barriers to distance education: A factor analytic study. The American Journal of Distance Education, 11(2), 39-54.

Müller, T. (2008). Persistence of women in online degree-completion programs. International Review of Research in Open and Distance Learning, 9(2), 1-18.

Nikolaki, E., Koutsouba, M., Lykesas, G., Venetsanou, F., y Savidou, D. (2017). The support and promotion of selfregulated learning in distance education. European Journal of Open, Distance and E-learning, 2O(1).

OECD (2012). How many young people graduate from tertiary education? In Education at a glance 2012: Highlights. OECD Publishing. doi: http://dx.doi. org/10.1787/eag highlights-2012-7-en

Osborn, V. (2001). Identifying at-risk students in videoconferencing and webbased distance education. American Journal of Distance Education, 15(1), 4154.

Park, J., y Choi, H. (2009). Factors influencing adult learners' decision to dropout or persist in online learning. Educational Technology \& Society, 12(4), 207-217.

Park, C. L., Perry, B., y Edwards, M. (2011). Minimising attrition: strategies for assisting students who are at risk of withdrawal. Innovations in Education and Teaching International, 48(1), 37-47.

Parker, A. (2003). Identifying predictors of academic persistence in distance education. United States Distance
Learning Association Journal, 17(1), 5561.

Pask, G. (1975). Conversation, cognition and learning. New York: Elsevier.

Perry, B., Boman, J., Care, W. D., Edwards, M., y Park, C. (2008). Why do students withdraw from online graduate nursing and health studies education? Journal of Educators Online, 5(1), 1-17.

Pierrakeas, C., Xeno, M., Panagiotakopoulos, C., y Vergidis, D. (2004). A Comparative Study of Dropout Rates and Causes for Two Different Distance Education Courses. The International Review of Research in Open and Distributed Learning, 5(2). doi: http://dx.doi.org/10.19173/irrodl. v5i2.183

Pigliapoco, E., y Bogliolo, A. (2008). The effects of psychological sense of community in online and face-to-face academic courses. International Journal of Emerging Technologies in Learning, 3(4), 60-69.

Pineda, C., Ortiz, A., y Moreno, I. (2001). Efectividad de las estrategias de retención universitaria: la función del docente. Educación y Educadores, 14(1), 119-135.

Prieto, O., y Duque, E. (2009). El aprendizaje dialógico y sus aportaciones a la teoría de la educación. Teoría de la Educación. Educación y Cultura en la Sociedad de la Información, 10(3), 7-30.

Roblyer, M. D. (2006). Virtually successful: Defeating the dropout problem through online school programs. Phi Delta Kappan, 88(1), 31-36.

Rodríguez-Muñiz, L. J., y Díaz, P. (2015). Estrategias de las universidades españolas para mejorar el rendimiento en matemáticas del alumnado de nuevo ingreso. Aula abierta, 43(2), 69-76.

Romero, C., y Ventura, S. (2010). Educational data mining: a review of the state of the art. IEEE Trans Systems Man Cybern Part C, 4O(6), 601-618.

Roso-Bas, F., Pades, A., y García, E. (2016). Emotional variables, dropout and 
academic perfomance in Spanish nursing students. Nurse Education Today, 37, 5358. doi: 10.1016/j.nedt.2015.11.021.

Rovai, A. P. (2003). In search of higher persistence rates in distance education online programs. The Internet and Higher Education, 6, 1-16.

Rué, J. (2014). El abandono universitario: variables, marcos de referencia y políticas de calidad. Revista de Docencia Universitaria, 12(2), 281-306.

Sánchez-Elvira, A. (2016). ¿Cómo desarrollar un Sistema de Apoyo al Estudiante de calidad en entornos mediados por tecnología? En M. Cruz y A. SánchezElvira (Eds.), Claves innovadoras para la prevención del abandono en instituciones de educación abierta y a distancia (195264). Santiago de los Caballeros: UAPAAIESAD.

Sánchez, A., y Elías, M. (2017). Los estudiantes universitarios no tradicionales y el abandono de los estudios. Estudios sobre Educación, 31(1), 27-48.

Scott, B. (2001). Gordon Pask's Conversation Theory: A Domain Independent Constructivist Model of Human Knowing. Foundations of Science, special issue on "The Impact of Radical Constructivism on Science, 6(4), 343-360.

Simpson, O. (2012). Supporting Students for Success in Online and Distance Learning. London: Routledge.

Simpson, O. (2013) Student retention in distance education: are we failing our students? Open Learning: The Journal of Open, Distance and e-Learning, $\quad 28(2), \quad 105-119, \quad$ doi: 10.1080/02680513.2013.847363.

Street, H. (2010). Factors Influencing a Learner's Decision to Drop-Out or Persist in Higher Education Distance Learning. Online Journal of Distance Learning Administration, 13(4).

Swan, K. (2001). Virtual interaction: Design factors affecting student satisfaction and perceived learning in asynchronous online courses. Distance Education, 22(2), 306331. doi: 10.1080/0158791010220208

Sun, S. Y. H. (2014). Learner perspectives on fully online language learning. Distance Education, 35(1), 18-42.

Tabbodi, M., Rahgozar, H., y Makki, M. (2015). The Relationship between Happiness and Academic Achievements. European Online Journal of Natural and Social Sciences 4(1), 241-246.

Tello, S. F. (2007). An analysis of student persistence in online education. International Journal of Information and Communication Technology Education, 3(3), 47-62.

Tinto, V. J. (1975). Dropout from higher education: a theoretical synthesis of recent research. Review of Educational Research, 45, 89-125.

Tladi, L. S. (2013). Factors affecting examination attrition: does academic support help? A survey of ACN203S (Cost Accounting and Control) students at Unisa. Open Learning: The Journal of Open, Distance and e-Learning, 28(1), 67-82.

Tresman, S. (2002).Towards a Strategy for Improved Student Retention in Programmes of Open, Distance Education: A Case Study from the Open University UK. International Review of Research in Open and Distance Learning, 3(1), 1-11.

Tuero, E., Cervero, A., Esteban, M., y Bernardo, A. (2018). ¿Por qué abandonan los alumnos universitarios? Variables de influencia en el planteamiento y consolidación del abandono. Educación $X X 1 ， 21(2)$, 131-154. doi: 10.5944/ educXX1.20066.

Vallejos, C. A., y Steel, M. F. (2017), Bayesian survival modelling of university outcomes. J. R. Stat. Soc. A, 18o, 613-631. doi: 10.1111/rssa.12211.

Vásquez, C., y Rodríguez, M. (2007). La deserción estudiantil en educación superior a distancia: perspectiva teórica y factores de incidencia. Revista 
Latinoamericana de Estudios Educativos, 37(3-4), 107-122.

Vygotsky, L. S. (1979). El desarrollo de los procesos psicológicos superiores. Barcelona: Crítica.

Wang, M. J. (2010). Online collaboration and offline interaction between students using asynchronous tolls in blended learning. Australasian Journal of Educational Technology, 26, 830-846.

Waugh, M., y Su-Searle, J. (2014). Student persistence and attrition in an online MS program: Implications for program design. International Journal on E-Learning, 13(1), 101-121.

Willging, P. A., y Johnson, S. D. (2004). Factors that influence students' decision to dropout of online courses. Journal of Asynchronous Learning Networks, 8(4), 105-118.

Wolters, C. (2003). Understanding procrastination from a self-regulated learning perspective. Journal of Educational Psychology, 95, 179-187.
Woodley, A., y Simpson, O. (2014). Student dropout: The elephant in the room. En O. Zawacki-Richter y T. Anderson (Eds.), Online distance education: Towards a research agenda, (459-484). Athabasca University Press: Athabasca.

Xenos, M., Pierrakeas, C., y Pintelas, P. E. (2002) A survey on student dropout rates and dropout causes concerning the students in the course of informatics of the Hellenic Open University. Computers \& Education, 39(4), 361-377.

Yasmin (2013). Application of the classification tree model in predicting learner dropout behaviour in open and distance learning. Distance Education, 34(2), pp. 218-231.

Zimmerman, T. (2012). Exploring learner to content interaction as a success factor in online courses. The International Review of Research in Open and Distributed Learning, 13(4), 152-165. doi: http:// dx.doi.org/10.19173/irrodl.v13i4.1302

\section{PERFIL ACADÉMICO Y PROFESIONAL DEL AUTOR}

Lorenzo García Aretio. Catedrático Emérito y Titular de la Cátedra UNESCO de Educación a Distancia. Ha sido decano de la Facultad de Educación de la UNED, director del Instituto Universitario de Educación a Distancia. Ha publicado 46 libros completos, 18 de ellos como autor único o autor principal, y los restantes como coordinador, director, editor o coautor. Cuenta con cuatro Doctorados Honoris Causa en cuatro países diferentes: Argentina, Colombia, Ecuador y México.

Email: lgaretio@edu.uned.es

Dirección:

Facultad de Educación (UNED)

Juan del Rosal, 14

28040 - Madrid - España

Fecha de recepción del artículo: 17/08/2018

Fecha de aceptación del artículo: 19/09/2018

Fecha de aprobación para maquetación: 27/09/2018 